\title{
PLEITOS RELACIONADOS CON EL USO DEL AGUA EN EL MONASTERIO ZARAGOZANO DE VERUELA DESDE SU FUNDACIÓN HASTA EL AÑNO 1400
}

\author{
Francisco Saulo Rodríguez Lajusticial
}

\section{RESUMEN}

El monasterio de Veruela fue el primer cenobio cisterciense de la Corona de Aragón. Durante su primer cuarto de milenio de vida, tuvo que atender a numerosos pleitos con otras instituciones que también pretendían el control de los valles del Moncayo. Entre los temas que causaban conflicto estaba el regadío y el uso del agua, preciado bien que, sobre todo a partir del siglo XIII, sería codiciado por las Órdenes Militares y por las villas de realengo instaladas en la región. Turnos de riego, recrecimiento de azudes, privilegios de unos para perjuicio de otros, etc. van a ser los grandes temas que vamos a encontrar a continuación.

Palabras clave: Veruela, Cister, Zaragoza, disputas, agua, Temple, Hospital, realengo.

\begin{abstract}
The monastery of Veruela was the first Cistercian abbey of the Crown of Aragon. During its first quarter of a millennium of life, monks had to respond to numerous lawsuits with other institutions that also claimed control of the valleys of Moncayo. Among the issues that caused conflict was irrigation and water use, precious good coveted, especially since the thirteenth century, by the Military Orders and royal small towns installed in the region. Irrigation shifts, construction of dams, privileges to a few to the detriment of others, and so on are going to be the main themes that we will find below.
\end{abstract}

Keywords: Veruela, Cistercian, Zaragoza, disputes, water, Temple, Hospital, royal properties.

$1 \quad$ Universidad de Zaragoza. Email: fsaulorodriguez@hotmail.com. 
Uno de los elementos clave que condicionó la instalación de toda comunidad cisterciense en un determinado lugar fue la existencia de cursos fluviales con cuya agua pudieran regarse las tierras que formaban parte de su dominio. En este sentido, como no podía ser de otra manera, el monasterio zaragozano de Veruela, el primero de toda la Corona de Aragón que albergó a monjes blancos, concedió al agua una importancia capital al organizar sus principales posesiones en las proximidades de ríos que aseguraran que no faltara este líquido vital. Esto trajo consigo un importante problema como fue el desarrollo de un abultado número de pleitos entre el cenobio y otras instituciones, ubicadas igualmente en las faldas del Moncayo, que necesitaban igualmente el agua para incentivar la producción en sus tierras.

Echando un vistazo a la documentación, a la hora de hablar de este tema nos encontramos, por un lado, con un siglo XII caracterizado por la adquisición a gran escala de derechos de riego a la par que se van incorporando nuevas tierras y por el desarrollo de toda una serie de acuerdos entre el monasterio y particulares y, por el otro, unos siglos XIII y XIV en los que se produjeron enfrentamientos mucho más crudos entre Veruela y las otras órdenes militares y/o pueblos del valle del Huecha.

\section{EL SIGLO XII: PACTOS Y ACUERDOS PARA EL USO DEL AGUA}

Tras la fundación del monasterio en el año 1146, nos encontramos con diversos documentos a lo largo del siglo XII que muestran a una comunidad cisterciense acordando con particulares o incluso con concejos el reparto del agua de un determinado cauce fluvial que casi siempre era el río Huecha o las acequias que bajaban de las cumbres del Moncayo.

Así, si la disponibilidad de agua fue un factor clave en cualquier punto del dominio monástico, lo fue todavía más si cabe en territorios en los que, por el tipo de cultivo, no se podía prescindir de este líquido. Éste es el caso, por poner un ejemplo, de los términos de Mazalcoraz y de Ginestar, en las inmediaciones de Magallón, en los que la alta concentración de viñedos hacía imprescindible la existencia de abundante agua que permitiera regarlos y en los que se produjeron varias donaciones de azumbres en los años sesenta y setenta de esta centuria. No por casualidad, éste sería el territorio en el que más conflictos se producirían por cuestiones relacionadas con el riego durante la Baja Edad Media.

De esta manera, el primer acuerdo en temas hídricos del que se tiene constancia data del año 1175 y se trata de un reparto del agua de Villamayor -en la actualidad, un despoblado que se situaba en las cercanías del municipio zaragozano de Bulbuente- que hicieron el monasterio y Gonzalo, de manera que los dos pudieran regar durante un día completo alternándose sin problemas $^{2}$.

Un acuerdo de mayor entidad en el que se vieron implicados varios pueblos del Moncayo se produjo en septiembre de 1179 en Borja, como consecuencia de las pesquisas realizadas por Blasco Maza de parte del rey Alfonso II sobre la distribución del agua de la acequia de Alfara que, por lo visto, era objeto de disputa entre el monasterio de Veruela y los hombres de Trasmoz.

\footnotetext{
2 Archivo Histórico Nacional (=A. H. N.), Códice 995B, fol. 82v.
} 
Resumiendo los detalles de un acuerdo del que ya hablaron Ángel González y Eusebio García ${ }^{3}$, el delegado real ordenó que los vecinos de Trasmoz fueran los primeros en aprovecharse del agua de Alfara por nacer allí la acequia (quod est Alfara mater de illa $a q u a^{4}$ ), teniendo derecho a regar durante tres días y tres noches, periodo tras el cual tomarían el agua los vecinos de Vera de Moncayo. Tras esto, una vez que el monasterio de Veruela hubiera regado todos sus campos y recolectado la cosecha (collectis suis messibus), se establece que el cenobio ya no pudiera aprovecharse más de esta acequia hasta el año siguiente.

Sin embargo, si interesaba el agua de las acequias del Moncayo, no menos la de la acequia mayor de Magallón que irrigaba la granja de Mazalcoraz, la principal de esta comunidad cisterciense. Dejando para más adelante los pleitos que se produjeron entre esta villa de realengo y los cistercienses, el copista del cartulario más antiguo conservado del cenobio -de finales del siglo XIII- ya destaca la importante cantidad de derechos de riego que Veruela había concentrado en este lugar: Trobase que ha la grangia de Maçalcorayg por las cartas de suso escriptas aqua en la cequia de Magallon XXXIII açumbres, e a otra parte un dia e una nueyt, menos de dos açumbres que fueron dadas en camio, según consta por la conveniencia de las cartas en do se tratará esta senyal ${ }^{5}$.

En el terreno de los acuerdos, es imprescindible citar el documento de 1 de junio de 1199 en el que los monjes pactaron el reparto del agua de Mazalcoraz con Farag de Alhacim, exarico de María de Agón, y sus hermanos. No entraré a detallar un documento un tanto extenso que consiste en la partición que se hizo del agua de riego entre este musulmán y los monjes en cada una de las propiedades -lo que permite conocer bien la microtoponimia magallonera de finales del siglo XII-, sino que únicamente dejaré constancia de un diploma que, como todos los anteriores, pretendió asegurar para el cenobio algo que le era fundamental para el desarrollo de sus cultivos.

\section{LOS SIGLOS XIII Y XIV: CONFLICTOS CON EL TEMPLE, EL HOSPITAL Y LA VILLA DE MAGALLÓN}

Al igual que sucedía con las disputas territoriales, los años comprendidos entre $1200 \mathrm{y}$ 1400 se caracterizaron por enfrentamientos mucho más crudos entre los distintos actores en juego, por lo que, como es fácil imaginar, el monasterio de Veruela tuvo que verse las caras con las órdenes militares con las que compartía las tierras del Moncayo, a saber, la del Temple hasta su disolución en la primera mitad del siglo XIV y la del Hospital, sin duda la gran heredera de los bienes de la primera.

3 A. GONZÁLEZ PALENCIA, "Notas sobre el régimen de riegos en la región de Veruela en los siglos XII y XIII", Al-Andalus, X, (1945), pp. 85-86 y E. GARCÍA MANRIQUE, Vera de Moncayo: un municipio del somontano ibérico, Zaragoza, Institución "Fernando el Católico", 1958, p. 75.

4 A. H. N., Códice 995B, fol. 35v. y A. H. N., Clero, Veruela, carp. 3764, doc. 8. Publica J. KIVIHARJU, Los documentos latino-romances del monasterio de Veruela 1157-1301: edición, estudio morfosintáctico y vocabulario, Helsinki, Suomalainen Tiedeakatemia, 1989, pp. 36-37.

5 A. H. N., Códice 995B, fol. 58r. Publica A. GONZÁLEZ, "Notas sobre el régimen de riegos en la región de Veruela en los siglos XII y XIII", citado, p. 87. 


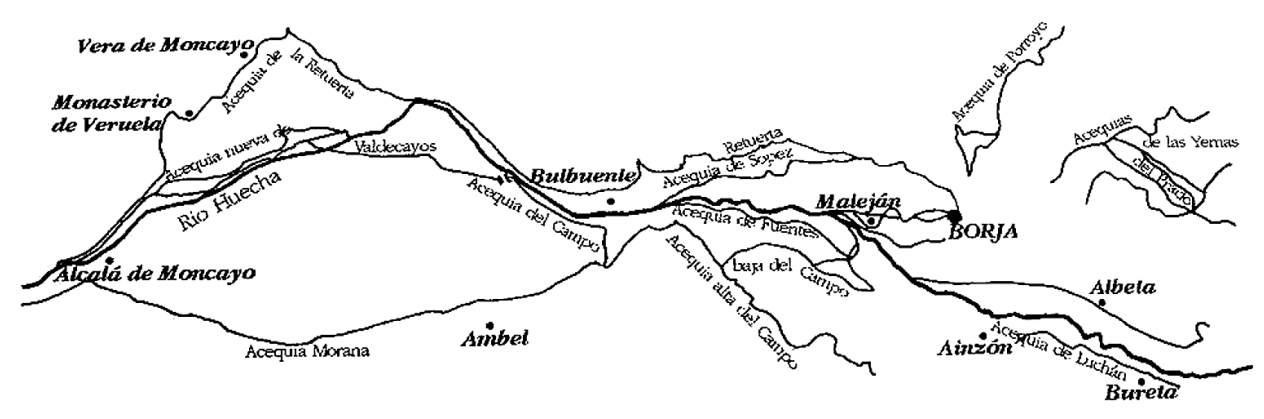

Esquema de los riegos del río Huecha

(Autores: Pedro Rújula y Herminio Lafoz ${ }^{6}$ )

El primer conflicto relevante se produjo en el año 1242 al producirse un desacuerdo entre el monasterio y los vecinos de Alcalá de Moncayo por un lado y el Temple y la encomienda de Ambel por el otro acerca del agua de la acequia de Morana. Resumiendo el conflicto, los ambeleros consideraban que Alcalá sólo tenía derecho a utilizar el agua de esta acequia entre los meses de abril y septiembre, mientras que los vecinos de Alcalá opinaban que debía hacerse extensivo este plazo a los meses de invierno por bever e por todas cosas que mester oviessen por la poblacion de Alcala ${ }^{7}$.

El conflicto se resolvió mediante la elección por cada una de las partes de representantes que fueran los encargados de decidir, compareciendo en este caso las autoridades de Ambel y de Alcalá, además de otras personalidades de importancia, como los comendadores de Novillas y de Ribaforada (Navarra) por parte de los templarios y el prior y cillerero mayor por parte de los de Veruela. Llama la atención que en esta negociación colectiva no estuviera presente el abad; sin embargo, esto se entiende si se tiene en cuenta que él, como máxima autoridad de la abadía, la representaba como parte litigante (y por consiguiente no podía encargarse de resolver el pleito), condición ésta que compartía con su rival, el maestre del Temple en Aragón y Cataluña.

Con todo, y pese a dar la sensación de que nos encontramos ante un duelo de titanes, el conflicto se resolvió con relativa sencillez al permitir los templarios que el monasterio de Veruela pudiera tomar también aguas de la acequia de Morana en los meses de invierno e incluso fer molinos en la dita cequia en lur terra, estableciéndose un sistema de turnos de riego entre ambas poblaciones que sería inviolable y que estaría controlado por zavacequias. No puede decirse que hubiera igualdad de condiciones puesto que los infractores de las normas pagaban de multa quinientos o mil quinientos sueldos jaqueses dependiendo de si eran de Ambel o de Alcalá, pero, en todo caso, está claro que el cenobio obtuvo un importante triunfo en este pleito.

No hay constancia de más enfrentamientos entre Veruela y Ambel por cuestiones re-

6 P. RÚJULA LÓPEZ y H. LAFOZ RABAZA, Historia de Borja. La formación histórica de una ciudad, Borja, Ayuntamiento de Borja, 1995, p. 88.

7 A. H. N., Códice $995 B$, fols. $114 \mathrm{v} .-115 \mathrm{v}$. 
lacionadas con el uso del agua a lo largo de toda la Edad Media. Sin embargo, a partir del siglo XVI las espadas volvieron a ponerse por todo lo alto en lo que se refiere a este tema, lo que llevó al autor de un Libro Registro del siglo XVIII a afirmar que los drechos que este monasterio tiene en la villa y territorio de Ambel se reducen al poder pacer con nuestros ganados en él y pasar el agua del río alto de Alcalá, llamada de Morana, por sus términos, y regar el nuestro de Villamaior. Y esto a costado tantos pleitos que desde el primero número asta el último todos son sentencias firmes ${ }^{8}$.

Cambiando de tema, así como el enfrentamiento de Veruela con Añón y Talamantes por el control de los valles del Moncayo fue el pleito más sonado de este periodo en lo que se refiere a límites territoriales, la controversia que se generó entre ambas instituciones, cistercienses y hospitalarios, a causa del recrecimiento de un azud fue también uno de los conflictos más importantes de la historia medieval del monasterio de Veruela en lo que se refiere a la gestión de sus recursos hídricos.

El problema consistía en un azud situado en Añón que, con el paso del tiempo, fue deteriorándose, lo que indignó a unos monjes de Veruela, interesados en su buen funcionamiento y en la necesidad de recrecerlo para administrar de forma eficaz las aguas que bajaban por la acequia de Morana. Así, este conflicto se caracterizó por los intentos continuados de Veruela de reparar el azud que siempre fueron bloqueados por los de Añón que, con el riego de sus cosechas asegurado, no estaban interesados en fomentar el auge del cenobio mediante esta reparación 9 .

El primer encontronazo por este tema se produjo en marzo de 1241 cuando los monjes empezaron a construir un ramal de acequia con el que distribuir mejor las aguas de la acequia de Morana, lo que motivó la rápida oposición de los hospitalarios de Añón que argumentaban que lo que hacían los monjes era una intromisión en un terreno que no les pertenecía. La clave pues de este primer pleito fue aclarar a quién pertenecía el azud, algo que ambos se disputaban ${ }^{10}$.

Encomendada la resolución del caso al justicia de Borja y al hermano del de Tarazona junto a dos personas más, requirieron documentación que acreditara la posesión del azud, algo que sólo pudo hacer Veruela al mostrar cartas del comte de Barçalona e del rey don Alfonso d'Aragon que certificaban que este azud pertenecía al monasterio de Veruela. Aunque esto en realidad no es del todo cierto puesto que en las donaciones que habían realizado Ramón Berenguer IV y Alfonso II en 1155 y en 1177 respectivamente se habla de los valles

$8 \quad M^{\mathrm{a}}$ D. CABANES PECOURT, El Libro Registro de Veruela, Zaragoza, Anubar, 1985, p. 144. Se trata de un libro elaborado en la segunda mitad del siglo XVIII en el que se comenta el conjunto de documentos que se guardaban en el monasterio durante esta centuria.

9 Pleitos de esta naturaleza tuvieron lugar en otros muchos monasterios cistercienses. Por poner un ejemplo, en 1328 el monasterio de Rueda se enfrentó a los mudéjares de Azaila porque éstos poseían un azud cuyo mal estado perjudicaba los intereses de este cenobio (cf. C. CONTEL BAREA, "El Cister zaragozano en los siglos XIII y XIV: abadía de Nuestra Señora de Rueda de Ebro. II. Documentos", Revista de Historia Jerónimo Zurita, 31-32, (1977), pp. 289-592).

10 En este documento se habla de açut de Trayt, lo que lo situaría en Alcalá de Moncayo y, por tanto, en tierras verolenses (cf. A. H. N., Códice 995B, fols. 87v.-88r. y A. H. N., Clero, Veruela, carp. 3766, doc. 15). Sin embargo, en pleitos posteriores relacionados con este mismo tema se dice claramente que el azud se encontraba en Añón, por lo que no sería exactamente el açut de Trayt. Da la sensación de que esto se trata de una consecuencia de que los monjes ganaran este pleito, lo que habría hecho al copista, el notario público de Alcalá, "acercar" dicho azud al lugar donde residía. 
del Moncayo en sentido general y no se dice nada de azudes ${ }^{11}$, lo cierto es que la existencia de certificados fue determinante y el monasterio se salió con la suya, aunque no por unanimidad al haber disentido de la sentencia uno de los jueces.

Obtenido este triunfo, las cosas parecieron calmarse hasta que volvieron a estallar las tensiones a comienzos del siglo XIV al querer impedir los de Añón los trabajos de reparación. Esta actitud provocó que el monasterio de Veruela pidiera auxilio a la monarquía, su tradicional aliada que en este caso estaba representada por un Jaime II que el año anterior había confirmado a los monjes la posesión de todos sus bienes y que en abril de 1304 les concedió licencia para reparar el azud sin interferencias de los vecinos de Añón ni del comendador. Esta autorización concedida por el rey fue publicada en abril de 1305 por el sobrejuntero de Tarazona, García López de Rueda, no sin que se produjeran protestas y apelaciones por parte de los de Añón ${ }^{12}$.

Sin embargo, pese a contar Veruela con el apoyo de la monarquía en este tema, poco o nada consiguió a efectos prácticos, puesto que a mediados de la primera mitad del siglo XIV la cuestión seguía tan paralizada como al principio. Así, en abril de 1330, una nueva sentencia arbitral promulgada por Pedro Juan de Mallén y Pedro Simón de Marchena volvía a denegar todas las apelaciones de los hospitalarios y volvía a autorizar a Veruela a que hiciera las obras de recrecimiento del azud de piedra o con estacas et en aquella manera que a ellos bien visto sera ${ }^{13}$, a cambio, eso sí, de indemnizar al comendador de Añón y a dos vecinos de esta localidad.

¿Qué se consiguió con esta nueva sentencia? Absolutamente nada, porque lo cierto es que el comendador de Añón hizo caso omiso de la misma y estorbó todo cuanto pudo, hasta el hecho de que, como en un cuento que nunca acaba, los monjes de Veruela volvieron a obtener en 1350 otra autorización del rey, en este caso Pedro IV, que de nuevo incidía en la libertad de los monjes verolenses para reparar el azud sin boicots por parte de los hospitalarios de Añón ${ }^{14}$.

Parece que en esta ocasión la comunidad cisterciense logró lo que tanto ansiaba, puesto que este tema ya no vuelve a salir a la luz hasta el año $1634^{15}$. En todo caso, lo visto aquí demuestra cómo, por un lado, la justicia no siempre era todo lo eficaz y rápida que hubiera sido deseable para los que se consideraban damnificados y cómo, por el otro, hubo siempre instituciones poderosas capaces de hacerle frente y que lograron cosas que nunca hubiera podido conseguir una simple aldea campesina. Esta oposición ejercida por los hospitalarios

11 Cf. para el documento de Ramón Berenguer IV, A. H. N., Códice 995B, fol. 36r. y Archivo de la Diputación Provincial de Zaragoza (=A. D. P. Z.), monasterio de Veruela, perg. 1. Publica V. DE LA FUENTE, España Sagrada, Madrid, Imprenta de José Rodríguez, 1866, tomo L, pp. 417-418.

Para Alfonso II, cf. A. H. N., Códice 995B, fol. 14 y A. H. N., Clero, Veruela, carp. 3764, doc. 2. Publican V. DE LA FUENTE, España Sagrada, citado, tomo XLIX, pp. 385-388; Ma D. CABANES PECOURT, "Los privilegios reales de Veruela en la segunda mitad del siglo XII”, Melanges Anselme Dimier, II, vol. 4, (1984), pp. 481-483 y A. I. SÁNCHEZ, Alfonso II rey de Aragón, conde de Barcelona y marqués de Provenza. Documentos (1162-1196), Zaragoza, Institución "Fernando el Católico", 1995, pp. 324-326.

12 A. H. N., Clero, Veruela, carp. 3770, doc. 10. El mandato real se encuentra inserto en este documento.

13 A. H. N., Clero, Veruela, carp. 3771, doc. 7.

14 A. H. N., Clero, Veruela, carp. 3773, doc. 2.

$15 \quad M^{\mathrm{a}}$ D. CABANES, El Libro Registro de Veruela, citado, p. 88. 
de Añón y por un comendador que desoía las órdenes del rey se sitúa además en uno de los momentos en los que el poder real estaba seriamente en entredicho con toda una serie de agrupaciones de nobles que le hicieron frente y que obtuvieron importantes logros; sin embargo, esto ya es otra historia.

Si hubo problemas por el riego entre los pueblos del Moncayo, fueron también frecuentes las fricciones entre el monasterio de Veruela y los pueblos de su dominio por un lado y la villa de Borja ${ }^{16}$ por el otro, en tanto en cuanto los borjanos debían regar con las mismas aguas que previamente pasaban por todas las tierras verolenses. En definitiva, como señaló Manuel Ramón Pérez, a lo largo de la historia se produjo un "tira y afloja" entre Veruela y Borja cuyo objetivo no era otro más que el control del valle del Huecha ${ }^{17}$.

Siendo una vez más la acequia de Morana la que llamaba la atención, a comienzos del siglo XIV había un cuádruple conflicto entre hospitalarios de Añón, templarios de Ambel, cistercienses y borjanos por el reparto de las aguas, sintiéndose éstos los más perjudicados de todos por el hecho de que eran los últimos a los que les llegaba al agua. Para arreglar el problema, intervino el propio monarca Jaime II, quien en agosto de 1303 modificó el turno de riegos vigente en la región que excluía a Borja, decretando que Ambel, Añón, Alcalá, Bulbuente y Villamayor disfrutaran del agua durante veinte días (repartiéndosela entre ellos como acostumbraban a hacer) y que cinco días fueran reservados para el riego por parte de los borjanos ${ }^{18}$.

Parece que el resultado no fue demasiado satisfactorio para las partes implicadas porque en los años treinta se produjo una situación de atasco tal que desembocó en un pleito tremendamente intrincado cuya sentencia está datada en mayo de 1335, si bien ya en 1334 estaba el ambiente caldeado a juzgar por que fue en este año cuando se eligieron a los representantes de cada uno de los pueblos, salvo el de Alcalá de Moncayo que ya había sido designado en 1332.

Este pleito, conservado en forma de copias al haberse perdido el original ${ }^{19}$, fue resuelto por el primer arzobispo de Zaragoza, Pedro López de Luna y Jiménez de Urrea, quien asignó a Borja derechos de riego los diez últimos días y noches de cada mes comprendido entre octubre y marzo, ambos inclusive (decem dies ultimos continuos cum decem noctibus uniuscuiusque diei in fine mensium octubris, novembris, decenbris, ianuarii, februarii et marcii a die festivitatis Santi Micaelis Archangeli mensis setenbris usque ad kalendas mensis aprilis ${ }^{20}$ ), sin que por ello el monasterio se viera privado en estos diez días del uso

16 En la actualidad ciudad, no alcanzaría esta condición hasta el siglo XV.

17 M. R. PÉREZ GIMÉNEZ, "Agua y pastos; luchas y alianzas por el control del valle de la Huecha entre la ciudad de Borja y el monasterio de Santa María de Veruela”, en El món urbà a la Corona d'Aragó del 1137 als decrets de Nova Planta. XVII Congrés d'Història de la Corona d'Aragó, S. Claramunt (coord.), Barcelona, 2003, pp. 737-753.

18 A. C. A., Real Cancillería, registro 186, fols. 250v.-251. Esta resolución de Jaime II ya fue destacada en A. GIMÉNEZ SOLER, "El problema de la variación del clima en la cuenca del Ebro", Memorias de la Facultad de Filosofía y Letras, I, (1922), p. 100.

19 En cuanto al original, está claro que debió de perderse a causa de su avanzado deterioro, tal y como informa el Libro Registro del siglo XVIII: Está esta sentencia en un pergamino grande y rollado, ya roto de puro viejo y de aver servido en los tribunales (cf. $\mathrm{M}^{\mathrm{a}} \mathrm{D}$. CABANES, El Libro Registro de Veruela, citado, p. 142).

20 A. H. N., Clero, Veruela, carp. 3774, doc. 16 (copia de noviembre de 1363) y Archivo Municipal de Borja (=A. M. B.), 2, 14, 210 y 210 bis (copia de diciembre de 1377). 
del agua para necesidades como cocinar o el cultivo de la huerta. En los restantes meses, el agua sería íntegramente de los concejos de Vera de Moncayo, Alcalá y Bulbuente para que procedieran a su administración como creyeran conveniente.

En el futuro, Borja se benefició de derechos de riego en acequias que discurrían por el dominio verolense, como es el caso del agua de la de Campo, que por cinco días en cada mes del año pertenecería a los borjanos según disposición de Pedro IV de marzo de 1360 o de la de la Retuerta, que fue entregada a éstos para su disfrute durante diez días mensuales entre septiembre y marzo, premio éste que concedía el rey por la parte activa que tuvo Borja durante la Guerra de los Dos Pedros ${ }^{21}$.

Por último, hay que decir que todas estas concesiones que arzobispo y reyes hicieron a los borjanos -y en las que el monasterio de Veruela se vio obligado a hacer muchas cesiones, no lo olvidemos- no debían de cumplirse demasiado bien en lo que se refiere a la acequia de la Retuerta, también llamada de Veruela (clamada de Beruela), puesto que en 1377 , tal y como he dicho antes, los borjanos solicitaron una copia de la sentencia arbitral promulgada por el arzobispo de Zaragoza en 1335. Sin duda los turnos no se debían de estar cumpliendo, pero tampoco tenemos más información a este respecto.

Siguiendo el curso del río Huecha, nos encontramos con lugares con los que el monasterio de Veruela, personificado en esta ocasión en la granja de Mazalcoraz, mantuvo grandes rivalidades por cuestiones relacionadas con el riego, a saber, con Alberite de San Juan (de nuevo los hospitalarios haciendo acto de presencia y, como se verá, a causa también de un azud) y, sobre todo, Magallón, villa de realengo con la que Veruela pleitearía bastante por los recursos hídricos durante toda la Baja Edad Media, tal y como destaqué en un artículo todavía no publicado ${ }^{22}$.

Comenzando por Alberite, el monasterio de Veruela tenía el mismo problema en este lugar que en Añón, es decir, la existencia de un azud en mal estado que se encontraba en dominio hospitalario provocaba que el caudal no fuera suficiente, por lo que constantemente había solicitado al comendador de este lugar que lo arreglara, obteniendo por lo general la callada por respuesta. Ante esta circunstancia, Magallón, Gañalur y Mazalcoraz se unieron a comienzos del siglo XIV para reparar el azud, tarea que en realidad no les correspondía hacer, por lo que Jaime II ordenó en noviembre de 1308 al comendador de Añón que costeara los gastos y que en adelante se comprometiera a mantener el azud en buen estado ${ }^{23}$.

Sin embargo, el grueso de los pleitos que tuvieron lugar en el siglo XIV -en las centurias anteriores no hay constancia de enfrentamientos- en este recodo del río Huecha se produjeron entre la granja de Mazalcoraz y Magallón, siendo el objeto de disputa el agua que discurría por la acequia mayor de este segundo lugar, ramal más o menos paralelo al recorrido del río Huecha cuyo recorrido explico con mayor detalle en mi artículo en prensa,

21 A. M. B., 2.13.207, 2.14.214 y 2.16.227 (copia de 1791). Estos documentos del Archivo Municipal de Borja fueron transcritos por Carmelo Rodríguez en su inédita tesis de licenciatura: C. RODRÍGUEZ ABAD, El señorío de Borja en la Edad Media, 3 vols. [tesis de licenciatura inédita]. Para una explicación del recorrido de todas estas acequías y del regadío del Huecha en general, cf. P. RÚJULA y H. LAFOZ, Historia de Borja. La formación histórica de una ciudad, citado, pp. 85-87.

22 F. S. RODRÍGUEZ LAJUSTICIA, "Pleitos entre Magallón y Mazalcoraz por cuestiones de riego en los siglos XIV y XV" [en prensa].

23 Archivo de la Corona de Aragón (=A. C. A.), Real Cancillería, Jaime II, registro 142, fol. 272v. 
tal y como también hace Simonne Teixeira en su tesis doctoral ${ }^{24}$ y como puede verse en el siguiente mapa:

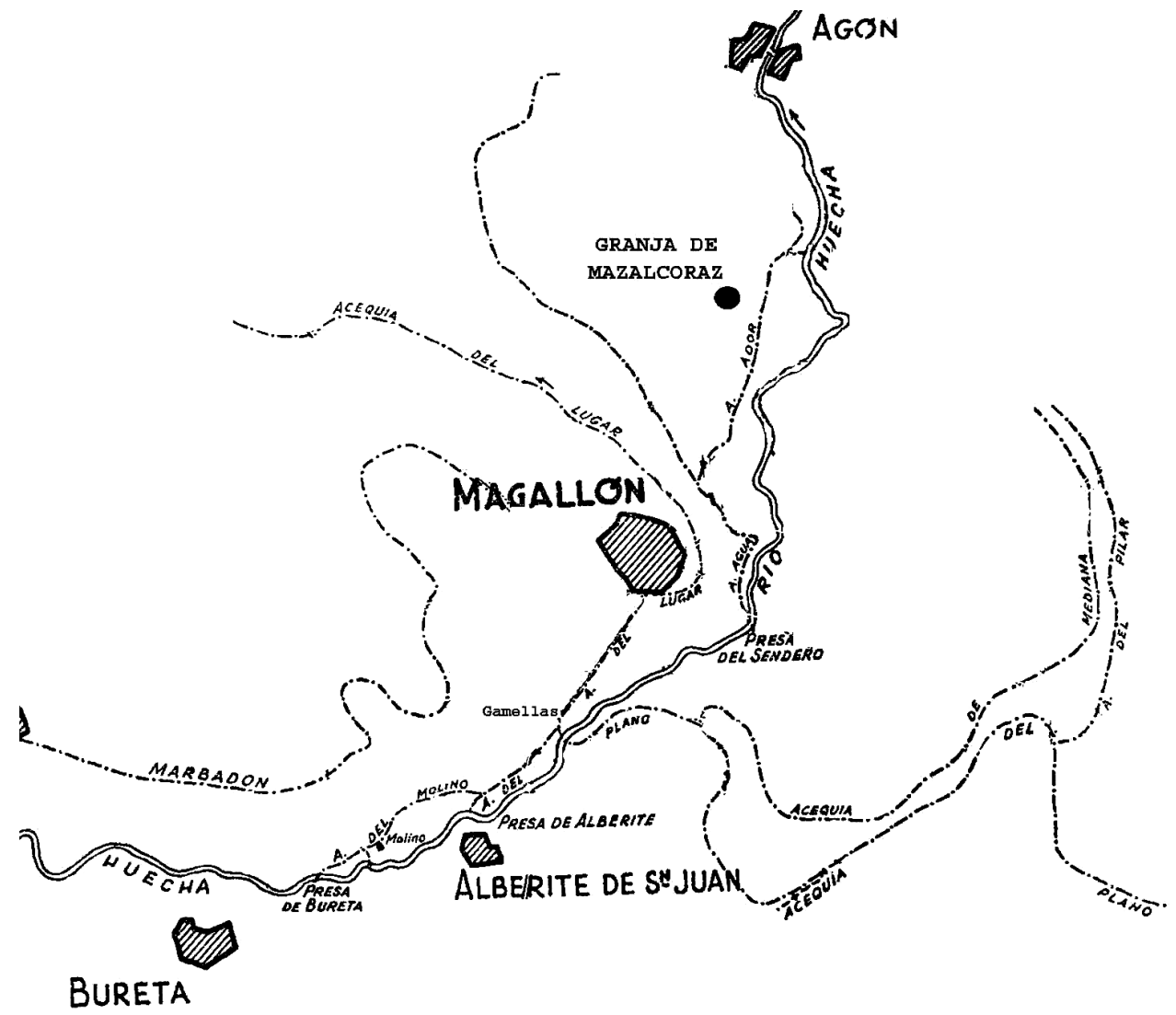

Río Huecha a su paso por Bureta, Alberite de San Juan, Magallón y Agón (Autor: Eusebio García Manrique ${ }^{25}$ )

Yendo a los principales detalles de los pleitos, el primero de ellos se produjo en junio de 1356 cuando, al igual que había sucedido en otras partes del dominio verolense, fue necesario establecer un sistema de turnos de riego que permitiera que tanto Magallón como Mazalcoraz pudieran aprovecharse del agua sin mayores tensiones. De esta manera, en esta concordia de 1356 se establece que el agua sea puesta en $v e z^{26}$ cada año para el primer

24 S. TEIXEIRA, El dominio del monasterio de Veruela: la gestión de un espacio agrario andalusí, pp. 323-326 [tesis doctoral en microficha].

25 E. GARCÍA MANRIQUE, Las comarcas de Borja y Tarazona y el Somontano del Moncayo. Estudio geográfico, Zaragoza, Institución "Fernando el Católico” e Instituto “Juan Sebastián Elcano”, 1960, p. 126.

26 Poner en vez equivale a establecer un turno, en este caso, para regar (cf. R. Ma CASTAÑER MARTÍN, Forma y estructura del léxico del riego en Aragón, Navarra y Rioja, Institución Fernando el Católico, Zaragoza, 1983, p. 55). 
domingo de abril, de manera que los monjes pudieran tomarla cuando les correspondiera sin ningún problema ni impedimento por parte de los de Magallón (que entren et puedan entrar luego en su augua en su vez los ditos abbat et convento, siquiere granja de Maçalcorach, et prender aquella sines de contrast et enbargo alguno ${ }^{27}$ ), permitiéndose también la posibilidad de organizar los turnos de riego antes del primer domingo de abril en caso de que algún año fuera necesario.

Por último, para acabar con esta cuestión, el tema de los turnos de riego se trata también en una nueva sentencia arbitral promulgada en noviembre de 1368, ratificando lo expresado en la de 1356, pero dando más detalles, lo que es una muestra de que las cosas no habían quedado (o no habían querido quedar) claras a los de Magallón y Mazalcoraz. Así, en este documento se amplía la información en cuanto al periodo en el cual se establecen turnos de riego y que, al parecer, comprendía desde el primer domingo de abril hasta el día 24 de junio, festividad de San Juan Bautista, algo que por otra parte era costumbre segunt que en los livros del tiempo pasado se contiene et y es constunbrado ${ }^{28}$.

Fuera de esos casi tres meses en los que había un turno de riego, el procedimiento era el siguiente: en primer lugar, el derecho de riego correspondía a los vecinos de Magallón (sian susanos et primeros en dreyto en regar sus heredades) y, una vez éstos habían satisfecho sus necesidades, entonces debían dejar que el agua discurriera por su cauce en dirección a Mazalcoraz no girandola a perdicion de manera que las heredades de la dita granja se puedan regar. Tras esto, una vez que las heredades de la granja habían quedado convenientemente regadas, el concejo de Magallón tenía potestad para vender el agua sobrante, pero consultando previamente al granjero y siempre y cuando allí no tuvieran necesidad de riego.

Es evidente que la necesidad de Mazalcoraz era un criterio muy subjetivo si la decisión se dejaba exclusivamente en las personas de allí, por lo que, previendo esta posibilidad, se decretó que en este caso se designaran dos personas, una por cada parte, de manera que fueran ellos los encargados de juzgar esta necesidad y obrar en consecuencia.

En definitiva, en esta sentencia de 1368, pese a esa preeminencia de Magallón a la hora de regar entre el 24 de junio y el 1 de abril, se ve perfectamente la tónica general que se adoptaría en el resto de disposiciones pertenecientes ya al siglo XV y que consistía en la adopción de un sistema de turnos en los periodos de mayor necesidad acuífera, siempre bajo la condición de que las necesidades de Mazalcoraz quedaran convenientemente cubiertas antes de emprender ningún tipo de operación con el agua.

Con posterioridad al año 1400, y en realidad durante todo su devenir histórico, el agua siguió dando quebraderos de cabeza a los monjes de Veruela, tanto en Mazalcoraz como en el monasterio en sí; sin embargo, estos casos escapan ya al periodo cronológico estudiado en este artículo.

27 A. H. N., Clero, Veruela, carp. 3773, docs. 13 y 14 (documento inserto) у A. H. N., Clero, Veruela, libro 18704, fols. 12-15.

28 A. H. N., Clero, Veruela, carp. 3776, doc. 17. 


\section{APÉNDICE DOCUMENTAL}

1350, abril, 24 Zaragoza

Pedro IV ordena al actual comendador de Añón y a los vecinos de dicho lugar que permitan al monasterio de Veruela la reconstrucción de un azud en dicho término tal y como se había decretado en tiempos de Jaime II, pese al incumplimiento del anterior comendador.

- A. H. N., Clero, Veruela, carp. 3773, doc. 2. Inserto en documento de 29 de abril de 1350.

Petrus, Dey ${ }^{29}$ gratia rex Aragone, Valencie, Maiorice, Sardinie et Corsice comesque Barchinone, Rosilionis et Ceritanie, fidelibus nostris comendatori qui nunc est vel fueryt pro tempore loci d'Annyon et hominibus loci ipsius, salutem et gratiam.

Clamosam peticionem nobis reverenter exybitta pro parte abatis et conventus monasterii Sancte Marie de Berueli, ordinis cisterciensis, vidimus continente quod quamquam ipsi et vasalli ipsius monasterii necnon homines concilii ville Burgie teneant et posideant ac tenuerint et posederint a magnis citra temporibus quoddam açutum situatum in termino dicti loci, comendator quod olim predecessor vester ipsius loci conaretur perturbare et inquietare predictos abatem et conventum nec non alios ius habentes in aqua ex dicto açuto vigore comisionis sibi facte per illustrisimum dominum Iacobum, felicis recordacionis avum nostrum, declararet per suam sentenciam dictos abatem ${ }^{30}$ et conventum et alios ius habentes in aqua ipsa fore in posesionem açuti iamdicti ipsumque construere pose (sic) ex lapidibus, si volerent, in termino loci predicti dictisque comendatori et hominibus ville iamdicte inibicionem fecisset quod dictum abatem et conventum in possesionem ipsius açuti aliquatenus non turbarent prout hec et alia in ipsa sentencia latius continetur.

Verumtamen, post magnum temporis spacium, comendator qui tunc erat ipsius loci contra tenorem sentenci iamdicte conatus fuyt turbare et inquietare abatem et conventum et alios ius habentes in açuto iamdicto et pro inde super questione ipsa tam parte (?) dicti abatis quam comendatoris impetum Johanis de Mallen, iurisperitum Tirasone, et Petrum Simonis de Marcell fuyt arbitraliter compromisum qui per suam sentenciam pronunciarunt et mandarunt dictum abatem et conventum et alios ius habentes in ea posse construere et facere er reparare dictum açutum ex lapidibus vel aliter prout eis melius exissteret expedire ut hec et alia in ipsa sentencia latius enarantur.

Et quamvis pretextu dictarum sentenciarum dictus abas et conventus et alii ius habentes in aqua predicta tenuerint et posederint ac teneant et possideant dictum açutum tamen vos nunc de facte potius quam de foro et racione conamini inquietare et perturbare memoratum $(\text { sic })^{31}$ abatem et conventum et alios ius habentes in aqua que ex dicto açuto sumitur

29 Es frecuente en este documento el uso de $y$ en vez de $i$.

30 Igualmente, siempre se utiliza la palaba abas en vez de abbas.

31 Si bien más adelante se repite esta misma expresión y allí se pone el adjetivo en plural (memoratos) como dictan las normas de la gramática latina, en esta ocasión se utiliza el singular aunque después vayan dos sustantivos. 
dubitentque ne per vos ipsum açutum dirivatur sehu etiam destruatur suplicarunt nobis propterea dictus abas et conventus et alii ius habentes in aqua iamdicta ut eis super hoc deberemus cum iusticia debita providere.

Qua suplicacione velut racioni consona recepta benigne, cum nobis costent de sentenciis antedictis de quibus in cancellaria nostra facta extitit plena fides fas esset litem ex lite prime materye consurgere, vobis dicimus et mandamus quatinus sentencias antedictas dicto abati et conventuy et aliis herentibus ius in qua (sic) açuti iamdicti observeris easque faciatis inviolabiliter observari iura ipsarum, continencias et tenores et super posesionem qua sunt eiusdem ipsos a modo minime perturbetis seu etiam inquietetis.

Aliter per presentes mandamus dilecto consiliario nostro iusticie Aragonis vel eius locum tenenti ut ordinario ceterisque oficialibus nostris presentibus et futuris quod ad tenendum et observandum contenta in sentenciis antedictis nec non manutendum et defendendum memoratos abatem et conventum et alios ius habentes in aqua ex açuto iamdicto et super possesionem qua sunt vos et quemlibet vestra compellant fortiter et districte prout de foro et recione extiteryt faciendum maliciis omnibus pretermissis.

Datum Cesarauguste, $\mathrm{VIII}^{\circ}$ kalendas madii anno Domini millesimo $\mathrm{CCC}^{\mathrm{o}}$ quinquagesimo. Exaravit Roma.

\section{II}

\section{0, abril, 29 Añón de Moncayo}

Los procuradores del monasterio de Veruela y de Vera de Moncayo muestran a las autoridades de Añón de Moncayo una carta del rey ordenándoles que no impidan la reconstrucción de un azud, comprometiéndose éstos últimos a no interferir.

- A. H. N., Clero, Veruela, carp. 3773, doc. 2.

Seppan todos que dia jueves, dos dias en la sallida del mes de abril era millesima CCC ${ }^{\mathrm{a}}$ LXXX $^{\mathrm{a}}$ VIII ${ }^{\mathrm{a}}$, en el castiello de Annyon, present mi, notario, et los testimonios de iusso scriptos, en presencia de los honrados honestos et religiosos don fray Arnalt de Bardaran, comendador; et fray Domingo Xemeno, prior de Annyon; et don Juhan Perez Ferrando, justicia de Annyon, fueron personalment constituydos don fray Gonçalvo, prior del monesterio de Santa Maria de Beruella, procurador que se dixo del abat et convento del dito monesterio, et Juhan de Navasa, procurador de los honbres del concello de Bera et presentaron a los ditos comendador et justicia huna karta en paper abierta et en el dorsso sellada con siello de cera amarilla del muyt alto et muyt excellent princep et sennyor don Pedro, por la gracia de Dios rey de Aragon, el tenor de la qual yes a tal:

\section{Inserta el documento anterior}

La qual, presentada et leyda, requirieron a los ditos comendador et justicia por ellos et en nombre de los hombres del concello d'Aynnon que ellos las cosas en la dita letra del sennyor rey contenidas obidiessen et exiguiessen en todas et por todas cosas et contra aquellas en alguna manera non viniessen nin venir fiçiessen; en otra manera que encorriesen 
las penas de las protecciones del sennyor rey en los quales eran los ditos abat et convento et los honbres del concello de Boria et las otras penas que encorrian et encorrer devian los crebantadores et traspasadores de los mandamientos del sennyor rey.

Et de las sobreditas cosas requirieron a mi, notario, que les ende fiçiese carta publica et los ditos comendador et justicia dixieron que la letra sobredita del dito sennyor rey recibian con devida reverencia et demandaron que les fues dada copia de aquella et ellos farian lo que deviesen.

Testimonios fueron desto presentes Juhan Perez de Vera, scudero veçino de Bulbuen, et Juhan Perez, vicario de Alcala.

Feyto fue esto dia, era et lugar de suso scriptos.

Et yo Miguel de Mallen, notario publico de autoridat del sennyor rey por todo el regno de Aragon, qui esta carta screvi et ray et mende aqui do se lie "por todo el regno de Aragon qui esta carta screvi et ray" et el mi sig(signo)no hy pus. 